\title{
Some Aspects of Simulations and Modeling in Relativistic Nuclear Collisions (Astro-Particle Physics)
}

\author{
Mohammad Ayaz Ahmad \\ Physics Department, Faculty of Science, University of Tabuk, Tabuk, Saudi Arabia \\ Email address: \\ mayaz.alig@gmail.com \\ To cite this article: \\ Mohammad Ayaz Ahmad. Some Aspects of Simulations and Modeling in Relativistic Nuclear Collisions (Astro-Particle Physics). \\ International Journal of High Energy Physics. Vol. 4, No. 5, 2017, pp. 58-64. doi: 10.11648/j.ijhep.20170405.12
}

Received: May 4, 2017; Accepted: September 13, 2017; Published: November 10, 2017

\begin{abstract}
In the present articles an attempt has been made for the determination of multiplicity fluctuations of the secondary charged particles produced in relativistic heavy ion collisions with the help of the Ginzburg-Landau (G-L) approach to find the first-order phase transition (QGP to hadron phase state). This study has been carried out for the experimental data along with the theoretical prediction of Ultra-relativistic Quantum Molecular Dynamics model (UrQMD) and Monte-Carlo (RanMC) simulation. The parameters of the theoretical model and the values of scaling exponent are found in good agreements.
\end{abstract}

Keywords: Multiplicity Distribution, Heavy Ion Collisions, Quark Gluon Plasma (QGP) Formation

\section{Introduction}

It is well-known that relativistic nuclear collisions by using heavy ion is an important tool to study possible creation of a hot new matter state, quark-gluon plasma (QGP) in laboratory. In such nuclear collisions, the QGP might be formed and the system will be cooling with expanding and undergo a phase transition from deconfined state (QGP) of matter to confined hadrons [1-2]. Since only the final particles in the collisions are observable experimentally one can search for signals about the phase transition from only those particles. The order of the phase transition is one of the basic thermodynamic characteristics $[3-7$ and there references in].

A schematic representation of the phase diagram of strongly interacting matter, showing the transition between hadronic matter and the quark-gluon plasma as a function of temperature and baryon chemical potential has been depicted in Figure 1 [8]. The phase diagram of strongly interacting matter gives the following informations:

(1) At low temperatures and baryon densities, the system can be described in terms of hadrons, nucleons, mesons and internally excited states of nucleons.

(2) In the high - temperature $\left(\sim 170 \mathrm{MeV}\right.$ or $\left.10^{12} \mathrm{~K}\right)$, high baryon density ( $\sim 5-10$ times density of nuclear matter) regions in which matter exists as a nuclear liquid, hadron gas, or quark-gluon plasma.
(3) The path followed by the early universe $[1,2,9]$ as it cooled from the quark-gluon plasma phase to normal nuclear matter is shown by an arrow on the left. The dotted line leading to a black rectangular spot near the bottom indicates the path taken by a neutron star [9] as it forms.

(4) Heavy-ion collisions follow a path between these two extremes, increasing both the temperature and baryon density. It may be verified in future experiments that the energy densities $\sim 1-3 \mathrm{GeV} / \mathrm{fm}^{3}$, equivalent to a temperature $T_{c} \sim 150-200 \mathrm{MeV}[1,2]$ or baryon density $\rho_{c} \approx 5-10$ times nuclear matter density can indeed be reached in heavy ion collisions.

A phase transition is said to be of first-order if there is at least one finite gap in the first derivatives of a suitable thermodynamic potential. A transition is said to be of secondorder if there is a power-like singularity in at least one of the second derivatives of the potential.

The characteristics of self-similar behavior in multiplicity fluctuations have also been found to exist in the frame-work of a theoretical model so called the Ginzburg-Landau (G-L) theory of second order phase transition [10]. The formalism for Ginzburg-Landau [10-13] and the scaling exponent in relation, $\beta_{q}=(q-1)^{v}$ help to provide a useful diagnostic tool to detect the existence of second order phase transitions in hydronization process. The parameters of this theoretical model have been found for both second-order [10-12], and first-order $[13,14]$ phase transitions and the scaled factorial 
moments and a universal scaling exponent $v$ were calculated.

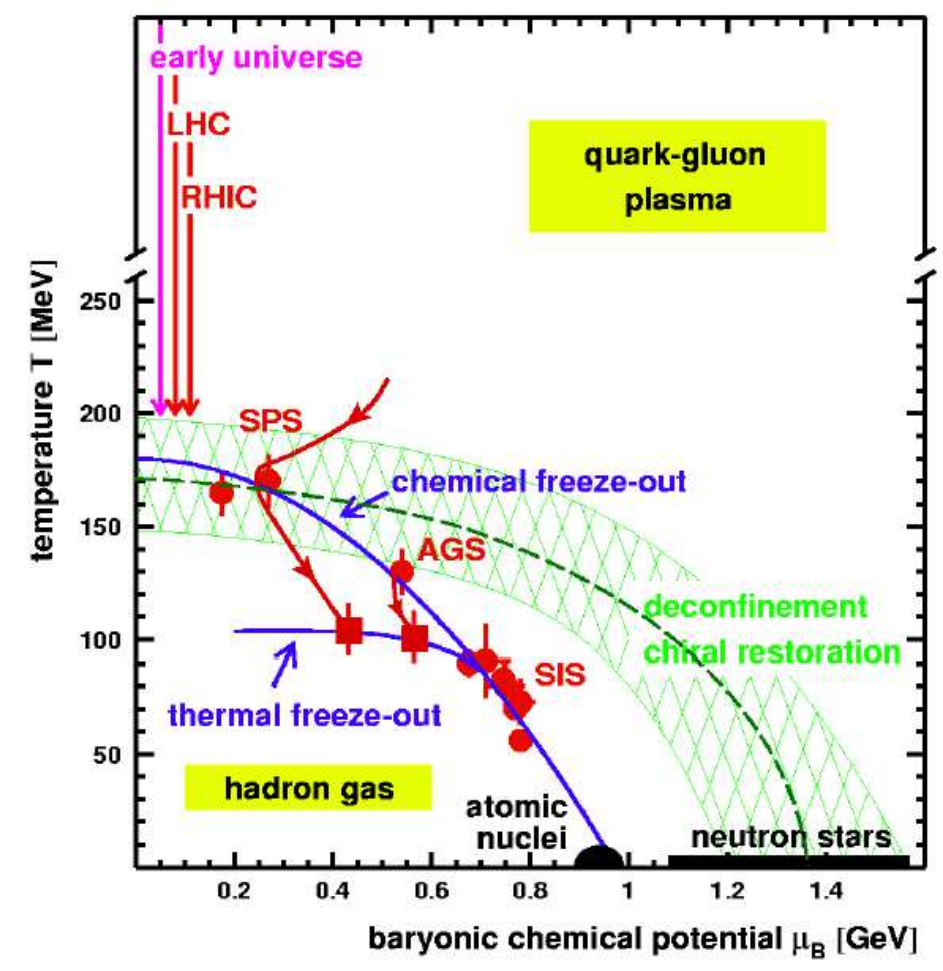

Figure 1. A schematic phase diagram of strongly interacting matter, showing the phase transition between hadronic matter and QGP as a function of temperature and baryonic chemical potential [8].

The Monte Carlo (MC) simulations on intermittency without phase transition for hadron-hadron ( $p-p)$ collisions give results different from theoretical predictions based on the G-L prediction [10].

In this article, we studied the difference between the scaling exponent " $v$ " which was calculated with the help of G-L model of QGP to hadrons phase transitions for Random events of Monte-Carlo simulations and the ultra-relativistic Quantum Molecular Dynamics model (UrQMD) [15, 16]. This simulation model was proposed specially to unearth the collision geometry of nucleus-nucleus interactions. There is no unique theoretical description to understand the dynamics of hadron-hadron collisions due to vastly different characteristics at different energies and in different kinematics intervals. The UrQMD describes the phenomenology of hadronic interactions at low and intermediate energies $(\sqrt{s}<5 \mathrm{GeV})$ in terms of interactions between known hadrons and their resonances. At higher energies, $\sqrt{s}>5 \mathrm{GeV}$, the excitation of color strings and their subsequent fragmentation into hadrons are taken into account in the UrQMD model. The UrQMD (version 1.3) is the most appropriate FORTRAN-77 based code, which is used for simulating heavy ion collisions in the energy range from SIS to RHIC and will be also used for future energy of PANDA and CBM experiments [16]. It has been designed as multipurpose tool for studying a wide variety of heavy ion related effects, ranging from multifragmentation and collective flow to particle production, fluctuations and correlations.

This simulation is based on the covariant propagation of all hadrons considered on the (quasi) particle level on classical trajectories in combination with stochastic binary scatterings, color string formation and resonance decay. It shows a Monte Carlo solution of a large set of coupled partial integral-differential equations for the time evolution of the various phase space densities of particle species $\mathrm{i}=\mathrm{N}, \Delta, \Lambda$, etc. The main ingredients of this model are the cross sections of binary reactions, the two body potentials and decay widths of resonances.

\section{Formulation of Ginzburg-Landau Model for Phase Transition}

The multiplicity distribution of produced charged particles in relativistic nuclear collisions for Ginzburg-Landau (G-L) theory is given such as following in the form of integral function [10-13].

$$
\mathrm{P}_{\mathrm{n}}=\mathrm{Z}^{-1} \int \mathrm{D}(\phi) \mathrm{P}_{\mathrm{n}}^{0} \mathrm{e}^{-\mathrm{F}(\phi)}
$$

where, $\mathrm{P}_{0}$ is the initial distribution for the pure state of $\phi_{0}$ in which the potential $F(\phi)$ has the minimum values such as:

$$
\mathrm{Z}=\int \mathrm{D}(\phi) \mathrm{e}^{-\mathrm{F}(\phi)}
$$

And the parameter $F(\phi)$ views for the Ginzburg-Landau free energy such as:

$$
\mathrm{F}\left(\phi=\int \mathrm{dz}\left\{\mathrm{A}|\partial \phi(\mathrm{z})|^{2}+\mathrm{B}|\phi(\mathrm{z})|^{2}+\mathrm{C}|\phi(\mathrm{z})|^{4}+\mathrm{D}|\phi(\mathrm{z})|^{6}\right\}\right.
$$

Where, $\phi$ is complex order parameter and it normalized on 
the mean multiplicity of produced charged particles in relativistic nuclear collisions with in the volume $\mathrm{V}$.

As it is demonstrated by other workers [3-7], the simplest form of the initial distribution $\left(\mathrm{P}_{\mathrm{n}}^{0}\right)$ will be the Poissonian distribution such as:

$$
\mathrm{P}_{\mathrm{n}}^{0}=\frac{1}{\mathrm{n} !} \exp \left\{-\int|\phi(\mathrm{z})|^{2} \mathrm{dz}\right\}\left(\int|\phi(\mathrm{z})|^{2} \mathrm{dz}\right)^{\mathrm{n}}
$$

Further, we establish the probability density distribution $\left(\mathrm{P}_{\mathrm{n}}^{0}\right)$ for the secondary charged pions produced in such nuclear collisions at high energies.

$$
\mathrm{P}_{\mathrm{n}}=\mathrm{Z}^{-1} \int \mathrm{D}(\phi) \frac{1}{\mathrm{n} !}\left(\int|\phi(\mathrm{z})|^{2} \mathrm{dz}\right)^{\mathrm{n}} \exp \left\{-\int|\phi(\mathrm{z})|^{2} \mathrm{dz}\right\} \mathrm{e}^{-\mathrm{F}(\phi)}
$$

Now the functional integration of Eqn. (5) is little bit hard. Therefore, we consider a simple case of uniform $\phi$ that is equivalent to setting $\mathrm{A}=0$ in eqn. (3). Then the integral function can be written such as:

$$
\mathrm{P}_{\mathrm{n}}=\mathrm{Z}^{-1} \int_{0}^{\infty} d x \frac{a x^{n} e^{-a x}}{n !} \mathrm{e}^{-x^{3}+b x^{2}+c x} \text { and } \mathrm{Z}=\int_{0}^{\infty} d x \mathrm{e}^{-x^{3}+b x^{2}+c x}
$$

where, the $x \sim|\phi|^{2}$, and the constants a, b, c can be determined by the fitting of experimental data.

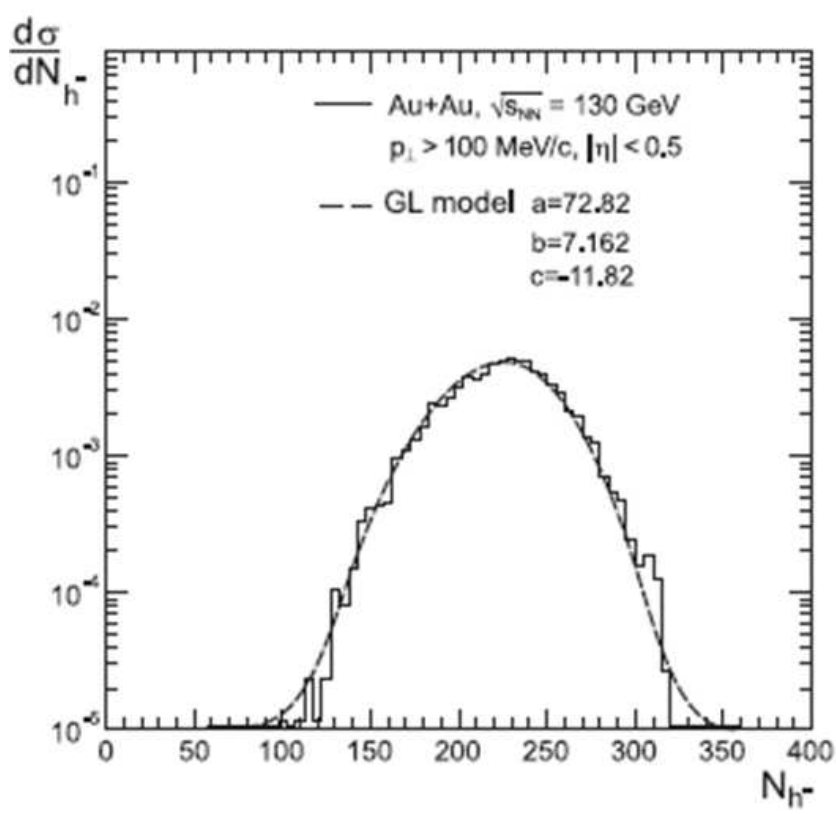

Figure 2. The multiplicity distributions of charged particles for heavy-ion $(A u+A u)$ collisions with Ginzburg-Landau $(G-L)$ parameters $a=72.82, b$ $=7.162, c=-11.82$ (dashed line) and experimental data(solid line).

The parameters of the Ginzburg-Landau (G-L) model for first order phase transitions at $(\mathrm{Au}+\mathrm{Au})$ collisions were determined in [17]. The findings of multiplicity distributions of secondary charged particles produced in the nuclear collisions of $(\mathrm{Au}+\mathrm{Au})$ were depicted in Figure 2. In this figure the Ginzburg-Landau (G-L) parameters $\mathrm{a}=72.82, \mathrm{~b}=$ $7.162, \mathrm{c}=-11.82$ respectively were represented with the dashed line and the experimental data with solid line. And both the findings were in a good agreement with other worker reported [10, 13-14, 17].

An efficient method for the study of multiplicity fluctuations of produced charged particles in relativistic nuclear collisions at ultra- high energies given by normalized factorial moments $\mathrm{F}_{\mathrm{q}}$ on the bin width $\delta$ of pseudo-rapidity ( $)$ such as $[18,19]$ :

$$
F_{q}=\frac{\langle n(n-1) \ldots \ldots(n-q+1)\rangle}{\langle n\rangle^{q}}=\frac{f_{q}}{f_{1}^{q}}
$$

where, $f_{q}=\langle n(n-1) \ldots \ldots(n-q+1)\rangle$ single event of normalized factorial moments, $\mathrm{n}$ is the number of secondary charged particle in small window ( $\delta$ ) of pseudo-rapidity $(\eta)$, all the average has taken over all events.

The power-law dependence of the normalized factorial moments on the number of bins represented by the above relation is known as intermittency [20-24]. Observation of such a power law may indicate a cascade mechanism of multiparticle production.

The power law predicts a characteristic linear rise of $\ln \left\langle F_{q}\right\rangle$ as a function of $\ln \delta$ which is represented by the following relation:

$$
\ln \left\langle F_{q}\right\rangle=\alpha_{q} \ln \delta+C
$$

Where, $\alpha_{q}$, which measures the strength of intermittency is called the intermittency exponent and $\mathrm{C}$ is a constant.

R. C. Hwa [22] has also obtained the following type of power law behavior:

$$
F_{q} \propto\left(F_{2}\right)^{\beta_{q}}
$$

Where, the values of $\beta_{q}$ summarize the scale invariance property on the global scale. The characteristics of selfsimilar behavior in multiplicity fluctuations have also been found to exist in the frame-work of the Ginzburg-Landau (GL) theory of second order phase transition [17].

Further, the relation (9) describes the relationship between $\mathrm{F}_{\mathrm{q}}$ and $\mathrm{F}_{2}$, irrespectively of their own dependence on $\delta$. If such property exists, the exponent $\beta_{q} \propto \frac{\ln F_{q}}{\ln F_{2}}$ should be approximately independent on $\delta$. And finally from relations (6) and (7) we get the followings:

$$
F_{q}=\delta^{q-1} \frac{\int_{0}^{\infty} d x(a x)^{2 q} \mathrm{e}^{-x^{3}+b x^{2}+c x}}{\int_{0}^{\infty} d x \mathrm{e}^{-x^{3}+b x^{2}+c x}}
$$

The scaling behavior is represented by the following relation:

$$
\beta_{q}=(q-1)^{v}
$$

The value of scaling parameter was predicted $(\boldsymbol{V}=1.304$ $\pm 0.35)$ as the critical exponent in the present experimental work for the parameters of Eqn. (3) $\mathrm{a}=72.82, \mathrm{~b}=7.162, \mathrm{c}=$ -11.82 respectively, where as it was found $(v=1.35 \pm 0.02)$ with some other workers [10, 13-14, 17]. 


\section{Results and Discussions}

In order to get more information about the production mechanism in high-energy nuclear collisions, the multiplicity distributions of the produced particles were studied. If the production of particles takes place independently of each other then multiplicity distribution represents Poisson distribution. On the contrary, if the production of a particle enhances the probability to produce other particles, then multiplicity distribution is broader than the Poisson's distribution and its normalized factorial moments are larger than 1 . The same thing should also happen for the multiplicity of particles produced in limited cells of the phase-space. It is believed that the multiplicity fluctuations in small phase-space bins can reveal important aspects of the multiparticle production mechanism such as intermittent pattern of fluctuations.

For the study of fluctuations in pseudo-rapidity $(\eta=-\ln$ $\left.\tan \left(\theta_{\mathrm{S}} / 2\right)\right)$ distributions, a given pseudorapidity interval of total length $\Delta \eta=\eta_{\max }-\eta_{\min }$, is divided into $\mathrm{M}$ bins of equal width, $\delta \eta=\Delta \eta / \mathrm{M}$. The scaled factorial moments, $\mathrm{F}_{\mathrm{q}}$, of order $\mathrm{q}$ is defined as:

$$
<F q>=\frac{M^{q-1}}{N_{e v}} \sum_{N_{e v}} \sum_{m=1}^{M} \frac{n_{m}\left(n_{m}-1\right) \ldots \ldots \ldots . .\left(n_{m}-q+1\right)}{<N>^{q}}
$$

where $\mathrm{n}_{\mathrm{m}}$ is the number of relativistic charged particles in the bin $\mathrm{m}^{\text {th }}(\mathrm{m}=1,2,3 \ldots \mathrm{M})$ and $<\mathrm{N}>$ represents the mean multiplicity of the hadrons in the pseudo -rapidity interval $\Delta \eta$ $=\mathrm{M} \delta \eta$.

The final findings from the present work have been depicted in Figures 3-6 respectively for relativistic nuclear collisions of $(\mathrm{Au}+\mathrm{Au})$ collisions at $\sqrt{\mathrm{SNN}}=130 \mathrm{GeV}$ along with the Random events of Monte-Carlo (RanMC) simulations and the ultra-relativistic Quantum Molecular Dynamics model (UrQMD) predictions. The predicted value of the scaling exponent $(v)$ was found of the order of $v=$
$1.304 \pm 0.35$ which was quite similar to the other workers [10, 13-14, 17].

In Figure 3, we draw the pseudo-rapidity distribution $(\mathrm{dn} / \mathrm{d} \eta)$ of produced charge particles in the nuclear collisions of $(\mathrm{Au}+\mathrm{Au})$ at an energy $\sqrt{\mathrm{SNN}}=130 \mathrm{GeV}$. This could be explained the geometry of such nuclear collisions as increase in the degree of centrality.

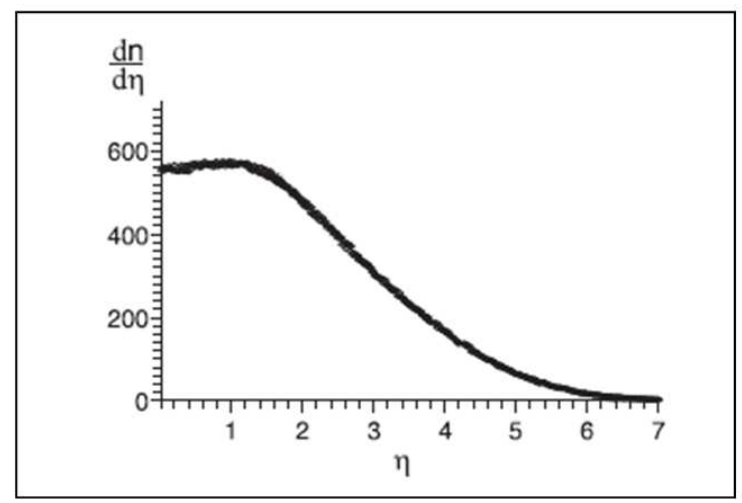

Figure 3. The pseudo-rapidity distribution $(d n / d \eta)$ of produced charged particles in the nuclear collisions of $(A u+A u)$ at an energy $\sqrt{ } S N N=$ $130 \mathrm{GeV}$.

In order to study the intermittency in multiparticle production, the whole pseudo-rapidity phase-space has been divided into number of bins $\mathrm{M}=2-30$ and the normalized factorial moment, $\mathrm{F}_{\mathrm{q}}$, for $\mathrm{q}=2-7$ are calculated by using Eqn. (12) due to the interactions caused by particles in the nuclear collisions of $(\mathrm{Au}+\mathrm{Au})$ at an energy $\sqrt{ } \mathrm{SNN}=$ $130 \mathrm{GeV}$. The dependence of $\ln F q$ on $-\ln \delta$ is shown in Figure 4 for these interactions. The linear rise in the values of $\ln \mathrm{F}_{\mathrm{q}}$ with $-\ln \delta$ in Figure 4, after a particluar value of $(-\ln \delta)$ indicates power law dependence of $\mathrm{F}_{\mathrm{q}}$ on the bins width $\delta$, which clearly suggests that an intermittent behaviour is being observed in these interactions.

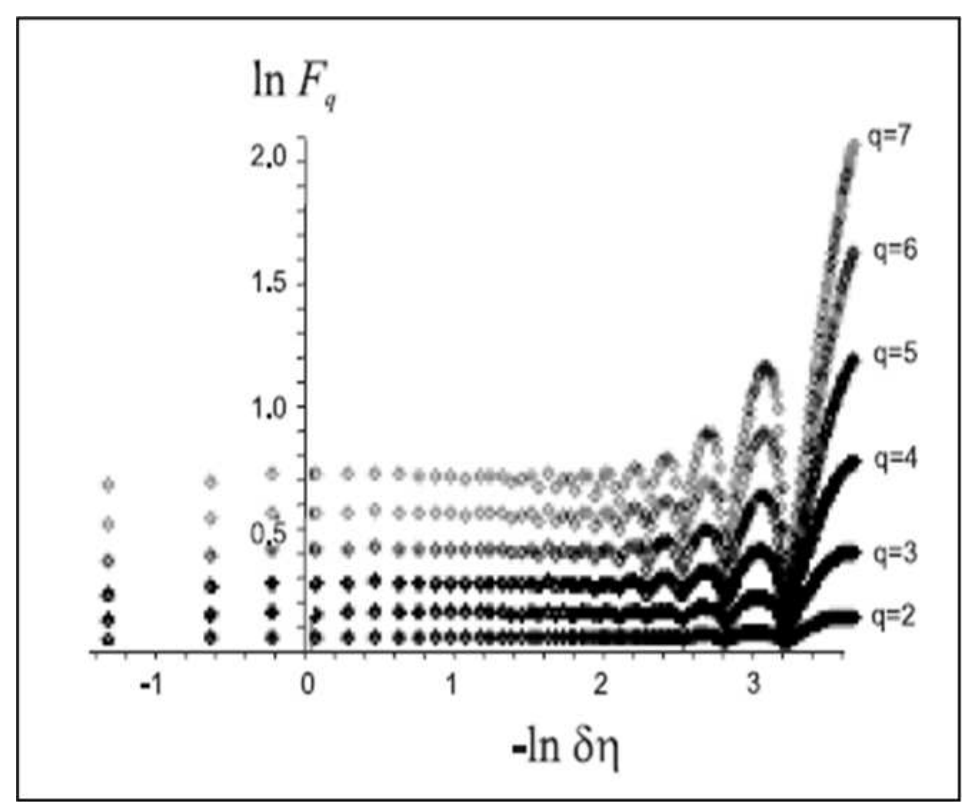

Figure 4. The dependence of $\ln F_{q}$ on $-\ln \delta$ for the order of moments $q=2-7$ of produced charged particles in the nuclear collisions of (Au + Au) at an energy $\sqrt{ }$ $S N N=130 \mathrm{GeV}$. 


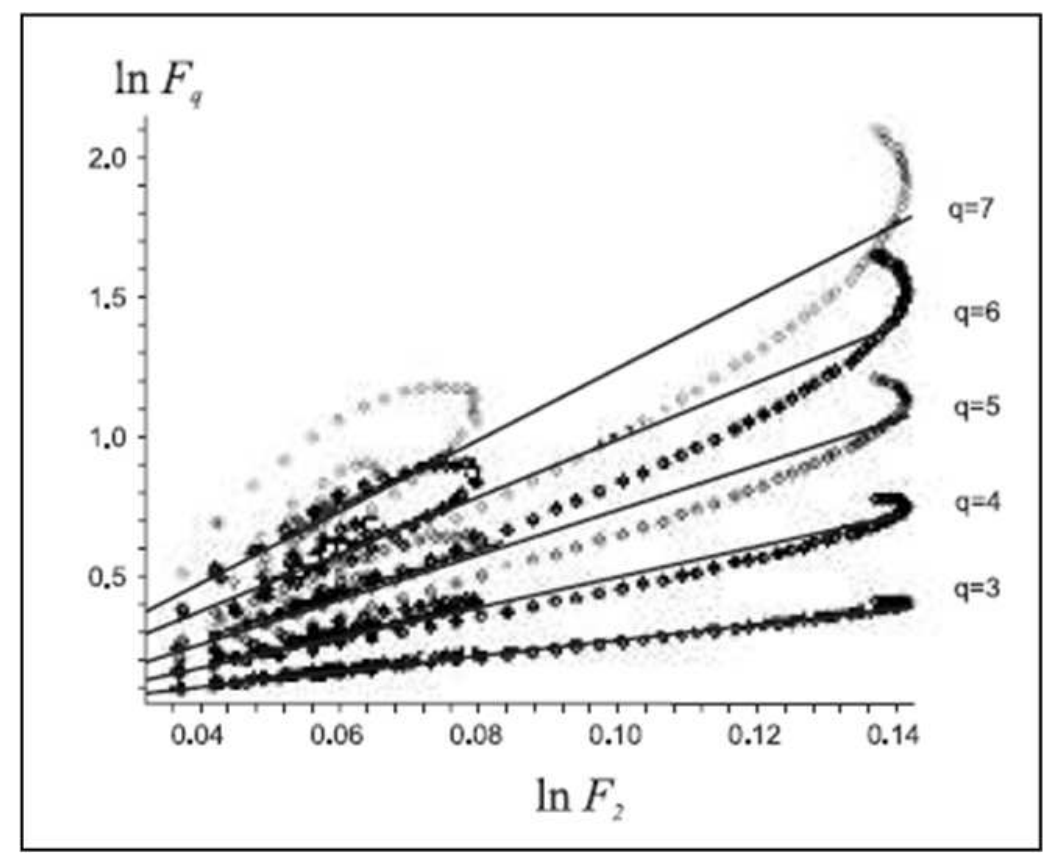

Figure 5. The variation of $\ln F_{q}$ on $\ln F_{2}$ for the order of moments $q=2-7$ of produced charged particles in the nuclear collisions of $(A u+A u)$ at an energy $\sqrt{ }$ $S N N=130 \mathrm{GeV}$.

The variation of $\operatorname{lnF}_{\mathrm{q}}$ as a function of $\operatorname{lnF}_{2}$ for each order of the moments has been shown in Figure 5. It is interesting to note from this Figure that a linear dependence of $\ln \mathrm{F}_{\mathrm{q}}$ on $\ln \mathrm{F}_{2}$ indicates the power law behavior, which confirms the validity of relation (9 and 11).

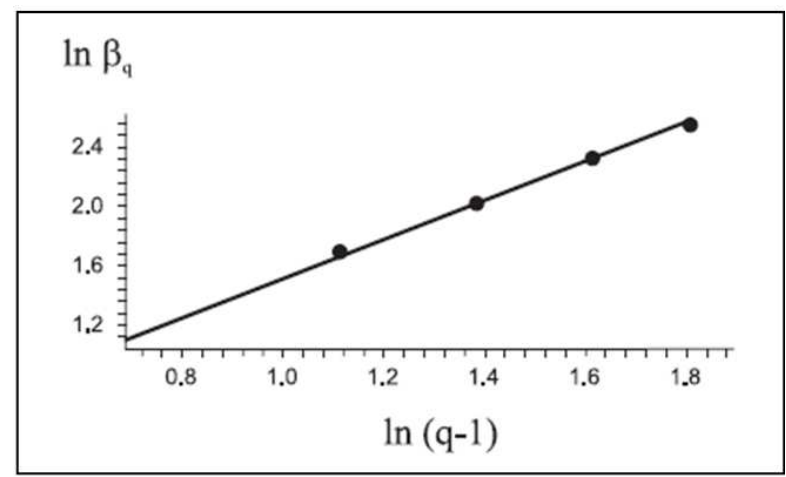

Figure 6. The dependence of $\ln \beta_{q}$ as a function of $\ln (q-1)$ of produced particles in the nuclear collisions of $(A u+A u)$ at an energy $\sqrt{ } S N N=$ $130 \mathrm{GeV}$.

The scaling exponent in above relation helps to investigate the existence of second order phase transitions in the hadronization process. If the experimental value of the scaling exponent, $v$, is less than the critical value of $1.304 \pm 0.35$, then the existence of a second order quarkhadron phase transition is expected [17]. The value of $\boldsymbol{V}$ higher than its critical value would imply the absence of such a phase transition. The experimental values of scaling exponent found in different experiments [10,13-14, 17] are more than its critical value. The dependence of $\ln \beta_{q}$ as a function of $\ln (\mathrm{q}-1)$ has been depicted in Figure 6. From this Figure it we find out that there is no clear evidence for the existence of a second order phase transition has been found in these experiments.

\section{Conclusions}

The relativistic heavy ion collisions provide an experimental setting for the study of the exotic behavior of the matter. Therefore one can concludes the followings remarks:

The Comparison of experimental results with the data generated using the ultra-relativistic quantum molecular dynamics (UrQMD) model reproduced similar pattern in most of the results. Further, it may be seen that the experimental data on intermittency exhibit a remarkable closeness to analogous data obtained from the UrQMD model. However, the MC generated events exhibit no such linear dependence on bins width $\delta$. This gives an indication for the absence of statistical contribution in experimental data. The flat behaviour in MC generated events is expected for independent emission of particles.

The higher experimental value of the scaling exponent, $v$, in comparison to the critical value indicates that no second order phase transition exists in the present interactions.

The experimental data presented in this article confirms that the nuclear geometry plays an important role in nucleusnucleus collisions at ultra-relativistic energies.

\section{Acknowledgements}

The author would like to acknowledge the keen support for this work of the Department of Physics, Faculty of Science, University of Tabuk, Saudi Arabia [25-31]. 


\section{References}

[1] Miklos Gyulassy. "Signatures of new phenomena in ultrarelativistic nuclear collisions." Nuclear Physics A418 (1984): 59-85.

[2] M. Stephanov, K. Rajagopal and E. Shuryak. "Event-by-Event fluctuations in heavy ion collisions and the QCD crititical point." - art. No. 114028. Physical Review D60 (11) (1999): 4028. DOI: 10.1103/PhysRevD.60.114028.

[3] R. C. Hwa, "Fractal measures in multiparticle production." Phys. Rev. D41, (1990): 1456-1462.

[4] R. C. Hwa, "Criticality, Erraticity and Chaoticity in Strong Interaction", Proc. $7^{\text {th }}$ Int. Workshop on Multiparticle Production, Correlation and Fluctuations, Nijmegen, Netherlands, (1996): 302.

[5] R. C. Hwa and Z. Cao, "Fluctuations of Spatial Patterns as a Measure of Classical Chaos." Phys. Rev. E 56, (1997): 326333.

[6] R. C. Hwa, Qing-hui Zhang, "Fluctuation of Gaps in Hadronization at Phase Transition." Phys. Rev. D62, 014003014009 (2000); nucl-th/0203022 (2002).

[7] Shafiq Ahmad and M. Ayaz Ahmad. "A comparative study of multifractal moments in relativistic heavy-ion collisions." Journal of Physics G: Nuclear and Particle Physics 32(9) (2006): 1279-1293.

[8] M. Ayaz Ahmad. (2010) A Study of Intermittency and Multifractality in ${ }^{28}$ Si-emulsion Collisions at $14.6 \mathrm{~A} \mathrm{GeV}$. [Ph. D. thesis], Aligarh Muslim University, Aligarh, India.

[9] J. D. Bjorken. "Highly relativistic nucleus-nucleus collisions: The central rapidity region." Physical Review D27 (1983): 140-151.

[10] R. C. Hwa, M. T. Nazirov, "Intermittency in second order phase transition." Phys. Rev. Lett. 69, (1992): 741 (1992).

[11] R. C. Hwa, J. Pan, Oregon University Report No. OITS/496, (1992).

[12] E. A. DeWolf, I. A. Dremin, W. Kittel, "Scaling laws for density correlations and fluctuations in multiparticle dynamics." Phys. Rep. 270, (1996), 1.

[13] Babichev, L. F. Non-Euclidean Geometry in Modern Physics: Proceedings of the 5th International Conference Boyai-GaussLobachevsky, Minsk, Belarus, October 10-13, (2006) / Minsk, (2006), 453.

[14] L. F. Babichev, D. V. Klenitsky, V. I. Kuvshinov. Proceed. III Int. Seminar "Nonlinear Phenomena in Complex Systems" (14-16 February, 1994, Polatsk, Belarus), Minsk, Institute of Physics, 1995, 149.

[15] S. A. Bass et. al., "Microscopic Models for Ultrarelativistic Heavy Ion Collisions." Progress Part. Nuclear Physics 41 (1998): 253-369.

[16] M. Bleicher, E. Zabrodin et. al., "Relativistic hadron-hadron collisions in the ultra-relativistic Quantum Molecular Dynamics model (UrQMD)." Journal of Phys. G: Nucl. \& Part. Phys., Vol. 25, (1999): 1859-1896.

[17] L. F. Babichev, A. N. Khmialeuski, and T. T. Chizhevskaya, "The Monte-Carlo Simulation of Heavy-Ion Collisions." Nonlinear Dynamics and Applications. Vol. 14 (2007) 20 24.

[18] A. Bialas, R. Peschanski, "Moments of rapidity distributions as a measure of short-range fluctuations in high-energy collisions." Nuclear Physics 273, (1986): 703-718.

[19] A. Bialas, R. Peschanski, "Intermittency in multiparticle production at high energy." Nuclear Physics, 308, (1988): 857-867.

[20] Shafiq Ahmad, M. Ayaz Ahmad, M. Irfan and M. Zafar. "Study of non-statistical fluctuations in relativistic nuclear collisions." Journal of the Physical Society of Japan, Vol. 75(6) (2006): 064604(1-9).

[21] Shafiq Ahmad and M. Ayaz Ahmad. "Some observations related to intermittency and multifractality in ${ }^{28} \mathrm{Si}$ and ${ }^{12} \mathrm{C}$ nucleus collisiond at 4.5A GeV." Nuclear Physics A780 (2006): 206-221.

[22] Shafiq Ahmad and M. Ayaz Ahmad, "Study of the levy stability and intermittent behavior in ${ }^{28} \mathrm{Si}$-emulsion collisions at 4.5A GeV." Nuclear Physics A, Vol. 789, (2007): 298-310.

[23] M. Ayaz Ahmad and Shafiq Ahmad, "Study of non-thermal phase transition in ${ }^{28} \mathrm{Si}$ - nucleus collisions at $14.6 \mathrm{~A} \mathrm{GeV}$." Int. Journal of Modern Physics E, Vol. 7 \& 8, (2007): 2241-2247.

[24] M. Ayaz Ahmad, Shafiq Ahmad and M. Zafar, "Intermittent and scaling behaviour of shower particles produced in the collisions of ${ }^{28} \mathrm{Si}-\mathrm{Em}$ at $14.6 \mathrm{~A} \mathrm{GeV}$." Indian J. of Physics 84 (12), (2010): 1675-1681.

[25] M. Tariq, M. Ayaz Ahmad, Shafiq Ahmad and M. Zafar. "Analysis of high $\mathrm{N}_{\mathrm{S}}$-multiplicity events produced in relativistic heavy ion collisions at $4.5 \mathrm{~A} \mathrm{GeV} / \mathrm{c}$." Romanian Reports in Physics Vol. 59(3) (2007): 773-790.

[26] M. Ayaz Ahmad, Mir Hashim Rasool and Shafiq Ahmad. "Scaling nature of target fragments in the interactions of ${ }^{28}$ Siemulsion at energy $14.6 \mathrm{~A} \mathrm{GeV}$." Ukrainian Journal of Physics. 58(10) (2013): 944-955.

[27] M. Ayaz Ahmad and Shafiq Ahmad. "Study of Angular Distribution and KNO Scaling in the Collisions of ${ }^{28} \mathrm{Si}$ with Emulsion Nuclei at 14.6A GeV." Ukrainian Journal of Physics. 57(12) (2012): 1205-1213.

[28] Shafiq Ahmad, M. Ayaz Ahmad, M. Tariq and M. Zafar, "Charged multiplicity distribution of relativistic charged particles in heavy ion collisions." Int. Journal of Mod. Physical E, Vol. 18 (9), (2009): 1929-1944.

[29] M. Ayaz Ahmad, Jalal Hasan Baker, "The Dependence of Average Multiplicity of Produced Charged Particles on Interacting Projectile Nucleons in Nuclear Collisions." DIALOGO JOURNAL, Vol. 3(1), (2016): 219-225.

[30] M. Ayaz Ahmad, "Some Aspects of Multi-Particle Productions in Relativistic Nuclear Collisions" DIALOGO JOURNAL, Vol. 3(1), (2016): 255-262.

[31] M. Ayaz Ahmad, "Intermittency Approach in the Nuclear Collisions of ${ }^{28} \mathrm{Si}+\mathrm{AgBr}$ at $14.6 \mathrm{~A} \mathrm{GeV}$ - Nuclear Emulsion Experiment a Particle Detector" European Journal of Interdisciplinary Studies, Vol. 7(2), (2017): 126-133. 


\section{Biography}

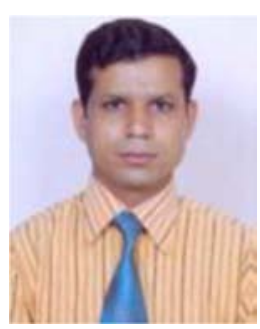

Mohammad Ayaz Ahmad is involved in teaching and research more than ten years. Besides the undergraduate courses $\mathrm{He}$ is teaching/taught courses of Nuclear Physics, Particle Physics and Electrodynamics to graduate / postgraduate students. For the past several years, He is working in the field of Experimental High Energy Heavy Ion Collisions Physics and has published research papers in various refereed journals, like Journal of Physics G (IOP Journal), Nuclear Physics A (Journal of Science Direct/ Elsevier Journals), Journal of Physical Society Japan, Internal National Journal of Mod. Physics E, Ukrainian Journal of Physics, e.t.c. 